\section{Evidence of upconversion luminescence contribution to the improved photoactivity of erbium doped $\mathrm{TiO}_{2}$ systems $\dagger$}

\author{
S. Obregón and G. Colón* \\ Received 10th May 2012, Accepted 18th June 2012 \\ DOI: $10.1039 / \mathrm{c} 2 \mathrm{cc} 33391 \mathrm{k}$
}

$\mathrm{Er}^{3+}-\mathrm{TiO}_{2}$ synthesized by a surfactant free hydrothermal method exhibits good photoactivities under sun-like excitation for the degradation of phenol. The presence of $\mathrm{Er}^{3+}$ does not affect the structural and morphological features of the $\mathrm{TiO}_{2}$ significantly. The best photocatalytic performance was attained for the samples with $2 \mathrm{wt} \%$ of Er. Different photocatalytic runs indicated that the incorporation of the $\mathrm{Er}^{3+}$ cation would be responsible for the enhanced photocatalytic activity, which participates in different mechanisms under UV and NIR excitation.

It is widely accepted that the photocatalytic technology based on semiconductor photoactive materials provides a feasible route to achieve two important processes for environmental control and producing energy, such as pollutant degradation and hydrogen production. ${ }^{1}$ Nowadays, the environmental problems demand increasingly severe regulations that open up opportunities for novel green photocatalytic routes leading to the alternative materials to traditional $\mathrm{TiO}_{2}{ }^{2}$ In order to improve the photocatalytic process, the utilization of solar light as efficiently as possible has been largely pursued. Thus, the use of visible light photons constitutes the key point for a good photocatalyst performance under sunlight conditions. For this scope, different strategies have been traditionally followed, in all cases for the improvement of visible photon absorption. Among these, doping of $\mathrm{TiO}_{2}$ with hybrid atoms or coupling of $\mathrm{TiO}_{2}$ with other semiconductors with low band gaps have proved to be viable ways to allow the extension of the light absorption edge. ${ }^{3-5}$ An alternative option consists of the creation of new single phase visible active catalysts which would overcome the drawbacks of doping. ${ }^{6-8}$

The other challenging composite configuration, rarely in use but currently under exploration, consists of the combination of a luminescence material with a photoactive catalyst. ${ }^{9,10}$ Within this configuration the exploitation of the radiation range not absorbed by the photocatalysts would be achieved. This approach appears to be a completely new alternative for

Instituto de Ciencia de Materiales de Sevilla, Centro Mixto Universidad de Sevilla-CSIC.C/Américo Vespucio, 49, 41092 Sevilla, Spain.E-mail: gcolon@icmse.csic.es; Fax: +34 954460665; Tel: +34954489536

$\dagger$ Electronic supplementary information (ESI) available: Experimental details and data concerning reaction, Raman and selected TEM images. See DOI: 10.1039/c2cc33391k enhancing the efficiency of the photocatalytic process by a wise handling of the arriving photons. It is clear that non-interacting photons drastically diminish the quantum yield under solar irradiation conditions. Thus, the combination of the photocatalytic system with the so-called phosphor material (formed by luminescent ions on a host matrix) which could handle and transform the incoming sunlight radiation turns out to be an interesting and not yet fully explored highway. The applications of the up-conversion process by phosphor-like systems (e.g. NIR or visible to UV) would optimize the photocatalytic performance of traditional UV active photocatalysts. ${ }^{11,12}$ Among various up-converting nanomaterials, $\mathrm{Er}^{3+}$-based compounds constitute an interesting option for this purpose which could be excited by NIR or visiblelight, showing luminescence in the visible and UV regions. ${ }^{13}$ Therefore, the assembly of $\mathrm{TiO}_{2}$ with such up-converting doping cations should in principle provide extra UV photons and increase the photocatalytic activity. In this communication, we propose the assembly formed by $\mathrm{Er}^{3+}$ doping ions and $\mathrm{TiO}_{2}$ presenting interesting high performance for phenol degradation under sun-like irradiation.

The systems were prepared following a hydrothermal method using a $\mathrm{TiO}_{2}$ colloidal solution as a $\mathrm{Ti}$ source and $\mathrm{Er}\left(\mathrm{NO}_{3}\right)_{3}$ (see $\mathrm{ESI} \dagger$ for details). After TEA addition (at $\mathrm{pH}=9$ ), the resulting suspension was transferred to a Teflon autoclave and subjected to a hydrothermal treatment at $140{ }^{\circ} \mathrm{C}$ for 20 hours. The obtained powder after filtration and drying was further calcined at $300{ }^{\circ} \mathrm{C}$ for 2 hours.

Regarding the crystalline structure, all photocatalysts, $\mathrm{Er}^{3+}-\mathrm{TiO}_{2}$, presented only the anatase phase (JCPDS 21-1272). No traces of $\mathrm{Er}_{2} \mathrm{O}_{3}$ were found even for higher doping loading (Fig. 1).

From XRD patterns, a slight diminution of the crystallite anatase size is observed, associated with the $\mathrm{Er}^{3+}$ incorporation on the crystalline structure. Inversely, BET surface area progressively increases as the $\mathrm{Er}^{3+}$ content increases (see Table S2 in ESI $\dagger$ for details). The $\mathrm{TiO}_{2}$ molar contents measured by EDX and XPS reasonably fit with the nominal values. This fact clearly indicates that $\mathrm{Er}^{3+}$ cations might be homogeneously accommodated within anatase structure. The Raman spectra of this series also support the presence of a single anatase phase (Fig. S1 in ESI $\dagger$ ). In addition to the anatase bands the corresponding fluorescence emission of $\mathrm{Er}^{3+}$ due to the excitation of a green laser $(532 \mathrm{~nm})$ can be detected in the Raman spectra. 


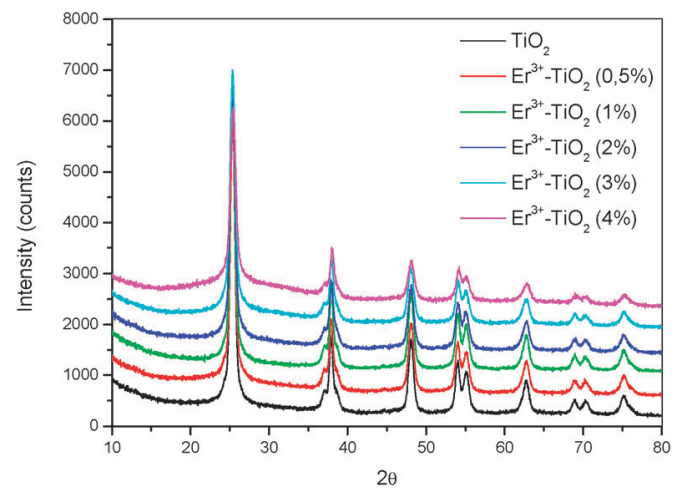

Fig. 1 XRD patterns of $\mathrm{Er}^{3+}-\mathrm{TiO}_{2}$ systems.

The morphology of the $\mathrm{TiO}_{2}$ catalysts appears to be slightly influenced by the presence of $\mathrm{Er}^{3+}$ (Fig. S2 in ESI $\dagger$ ). The synthetic route used for the preparation of photocatalysts produces a homogeneous distribution of round particles with sizes below $15 \mathrm{~nm}$ in all cases. As the $\mathrm{Er}^{3+}$ content increases the average size progressively decreases reaching a value of ca. $10 \mathrm{~nm}$ for $4 \mathrm{at} \% \mathrm{Er}^{3+}$. These values are in good agreement with crystallite size obtained from XRD patterns.

The calculated band gaps of $\mathrm{Er}^{3+}-\mathrm{TiO}_{2}$ appear to be similar to those of $\mathrm{TiO}_{2}$, being at around $3.2 \mathrm{eV}$. Therefore, the presence of erbium cations does not affect the band structure of anatase significantly.

The conversion plots for phenol degradation plainly show that doped photocatalysts exhibit higher performances than the pristine systems till erbium loading reaches 2 at\% (Fig. 2). This photocatalytic behavior of the composite structures clearly denotes a synergistic effect between $\mathrm{Er}^{3+}$ and $\mathrm{TiO}_{2}$.

By observing the reaction rates calculated from the conversion plots (see Table S2 in ESI $\dagger$ ), it is evident that along the series the sample with better performance corresponds to $\mathrm{Er}^{3+}$ doping of 2 at $\%$, decreasing the reaction rates progressively as the dopant content increases.

In order to discuss the particular contributions of each region of lamp emission, different photocatalytic runs were performed. Thus, by excluding the NIR contribution similar evolution of the reaction rate with respect to the UV-vis-NIR experiment was obtained (see Table S3 in ESI $\dagger$ ). It must be indicated that though similar evolution is found, the reaction rates under these conditions appear to be lower than those

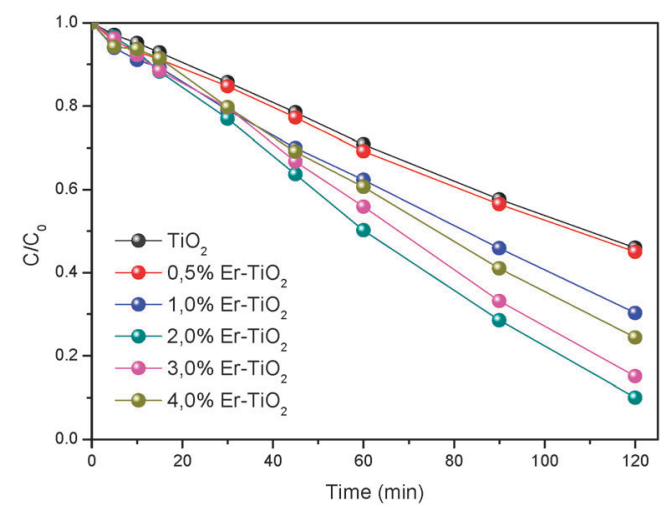

Fig. 2 Phenol degradation conversion plots for $\mathrm{Er}^{3+}-\mathrm{TiO}_{2}$ systems under sun-like excitation.
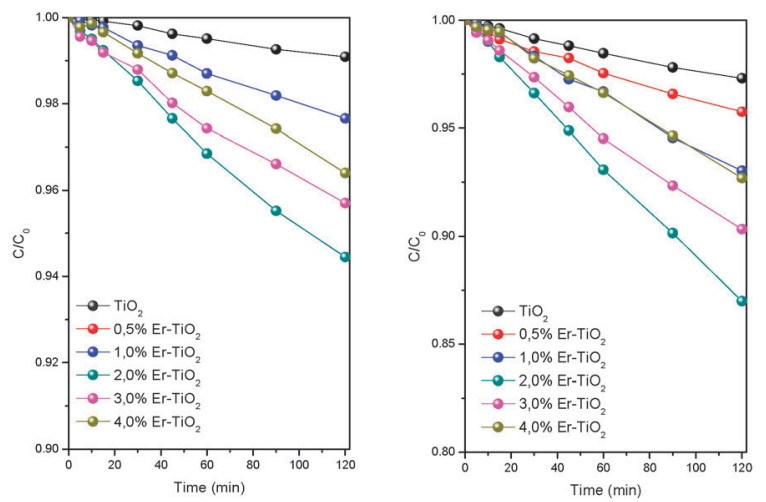

Fig. 3 Conversion plots for $\mathrm{Er}^{3+}-\mathrm{TiO}_{2}$ systems under vis-NIR excitation for (a) phenol and (b) methylene blue degradation.

under UV-vis-NIR, even assuming the diminution in the UV due to the IR filter. Moreover, by excluding the UV and NIR regions, no photocatalytic effect was observed under visible excitation. Therefore, it might be inferred that an improvement effect due to $\mathrm{Er}^{3+}$ doping was observed under UV irradiation. The evolution of the conversion plots shows that all doped $\mathrm{TiO}_{2}$ catalysts exhibit photoactivity for phenol degradation without UV irradiation (Fig. 3).

This photoactivity increases as the $\mathrm{Er}^{3+}$ content reaches a value of 2 at $\%$.

Similarly, we have performed the degradation of methylene blue under the same irradiation conditions and we have stated that the same trend is found.

On the basis of the presented results, two possible pathways might be considered for the generation of charge carriers (Scheme 1). On the one hand, under UV excitation, the clear improvement of the photoactivity would be related to the electron scavenger role of $\mathrm{Er}^{3+}$. Within this step $\mathrm{Er}^{3+}$ could be reduced to $\mathrm{Er}^{2+}$ by trapping the photogenerated electron. This $\mathrm{Er}^{2+}$ species would participate in the $\mathrm{O}_{2}$ reduction reaction. This way the presence of $\mathrm{Er}^{3+}$ would induce an effective charge carrier separation.

Indeed, it has been reported that in lanthanide doped $\mathrm{TiO}_{2}$ there is an important role of the traps in the transit of the electrons in the doped systems. ${ }^{14,15}$ Betenelli et al. argued that a small fraction of the trapping-detrapping process would be responsible for the lowering of diffusion coefficient. ${ }^{14}$

On the other hand, upon NIR irradiation, the presence of $\mathrm{Er}^{3+}$ cations would promote an upconversion process pumping photons in the UV range into the $\mathrm{TiO}_{2}$ structure. It has been widely reported that $\mathrm{Er}^{3+}$ shows $\mathrm{UV}$ photoluminescence emission between 390 and $410 \mathrm{~nm}$ after excitation at $980 \mathrm{~nm} .{ }^{16-18}$ This up-conversion process would involve a sequential three photon absorption $\left({ }^{4} \mathrm{I}_{15 / 2} \rightarrow{ }^{4} \mathrm{I}_{11 / 2},{ }^{4} \mathrm{I}_{11 / 2} \rightarrow{ }^{4} \mathrm{~F}_{7 / 2}\right.$ and $\left.{ }^{4} \mathrm{~S}_{3 / 2} \rightarrow{ }^{2} \mathrm{G}_{7 / 2}\right)$. Then, by a multiphonon relaxation the ${ }^{2} \mathrm{G}_{7 / 2}$ excited state decays to ${ }^{2} \mathrm{G}_{11 / 2}$ and ${ }^{2} \mathrm{H}_{9 / 2}$ lower states. The photoluminescence emission in the $\mathrm{UV} /$ violet range is then produced by the ${ }^{2} \mathrm{G}_{11 / 2} \rightarrow{ }^{4} \mathrm{I}_{15 / 2}$ transition, giving small emissions at 390 and $406 \mathrm{~nm} .{ }^{17,18}$ Thus, it can be assumed that the improvement of the photoefficiency might be related to the increasing number of available photons with the appropriate energy.

The obtained photocatalytic results clearly evidenced that the introduction of luminescent material into the $\mathrm{TiO}_{2}$ matrix 
(a)
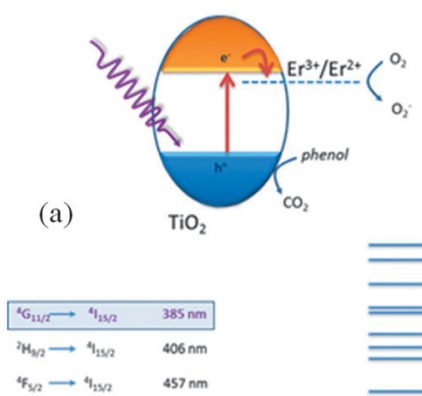

(b)

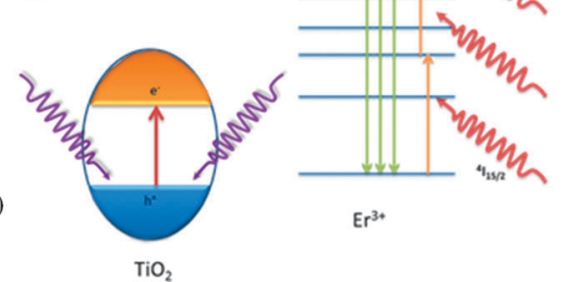

Scheme 1 Mechanism of the photocatalytic induction process in the $\mathrm{Er}^{3+}-\mathrm{TiO}_{2}$ heterostructures: (a) UV-assisted mechanism and (b) NIR upconversion assisted mechanism.

would promote the profiting of NIR photons, enhancing the photoactivity of the catalyst. This way our material opens up a wide roadway for the development of an integral solar active photocatalyst by the appropriate handling of the incoming photons.

This work was supported by the projects ENE2011-24412 and $P 09-F Q M-4570$. S. Obregón thanks CSIC for the concession of a JAE-Pre grant. We also acknowledge Dr Hernán Míguez for fruitful discussion of the results.

\section{Notes and references}

1 G. Colón, C. Belver and M. Fernández-García, Nanostructured Oxides in Photocatalysis, in Synthesis, Properties and Application of Oxide Nanoparticles, ed. M. Fernández-García and J. A. Rodríguez, Wiley, USA, 2007, ch. 17, (ISBN: 978-0-471-72405-6).

2 A. Kubacka, M. Fernández-García and G. Colón, Chem. Rev., $2012,112,1555$.

3 A. Kubacka, B. Bachiller-Baeza, G. Colón and M. FernándezGarcía, J. Phys. Chem. C, 2009, 113, 8553.

4 M. C. Hidalgo, M. Maicu, J. A. Navío and G. Colón, J. Phys. Chem. C, 2009, 113, 12840.

5 G. Colón, S. Murcia López, M. C. Hidalgo and J. A. Navío, Chem. Commun., 2010, 46, 4809.

6 X. C. Song, Y. F. Zheng, R. Ma, Y. Y. Zhang and H. Y. Yin, J. Hazard. Mater., 2011, 192, 186.

7 S. I. Naya, M. Tanaka, K. Kimura and H. Tada, Langmuir, 2011, 27, 10334.

8 S. Obregón, A. Caballero and G. Colón, Appl. Catal., B, 2012, 117-118, 59.

9 Z. Zhang, W. Wang, J. Xu, M. Shang, J. Ren and S. Sun, Catal. Commun., 2011, 13,31.

10 T. Zhou, J. Hu and J. Li, Appl. Catal., B, 2011, 110, 221.

11 W. Qin, D. Zhang, D. Zhao, L. Wang and K. Zheng, Chem. Commun., 2010, 46, 2304.

12 T. Li, S. Liu, H. Zhang, E. Wang, L. Song and P. Wang, J. Mater. Sci., 2011, 46, 2882.

13 A. J. Kenyon, Prog. Quantum Electron., 2002, 26, 225.

14 M. Betenelli, A. Speghini, D. Falcomer, M. Daldosso, V. Dallacasa and L. Romanó, J. Phys.: Condens. Matter, 2006, 18, S2149.

15 H. Liu, T. Peng, D. Ke, Z. Peng and C. Yan, Mater. Chem. Phys., 2007, 104, 377.

16 G. Wang, W. Qin, J. Zhang, J. Zhang, Y. Wang, C. Cao, L. Wang, G. Wei, P. Zhu and R. Kim, Opt. Mater. (Amsterdam), 2008, 31, 296.

17 X. Wang, G. Shan, K. Chao, Y. Zhang, R. Liu, L. Feng, Q. Zheng, Y. Sun, Y. Liu and X. Kong, Mater. Chem. Phys., 2006, 99, 370.

18 Q. Huang, J. Yu, E. Ma and K. Lin, J. Phys. Chem. C, 2010, 114, 4719 . 